\title{
20 BIOLOGICAL ROLES OF POLYNUCLEAR IRON(III)
}

Although iron metabolism has been extensively studied throughout the history of medicine, the importance of chelation and polymerisation of iron(III) to an understanding of iron biochemistry has been realised only recently. In contrast to the metabolism of other metals, most of the body iron is continuously recycled. Since very little iron is lost via urine, faeces and sweat, control of the iron level present occurs by regulation of uptake, not of excretion. Environmental iron that is presented to organisms as insoluble iron(III) hydroxide is biologically unavailable. A primary role of dietary chelating agents is to maintain iron(III) in a low molecular weight soluble form that can be utilised for absorption across the intestinal mucosa. Macromolecular chelates of iron(III), which compete with low molecular weight chelates for dietary iron and are resistant to the proteolytic enzymes in the gut, are expected to inhibit iron absorption. This inhibition becomes increasingly important as the molar ratio of iron to protein increases. In metalloproteins containing many iron(III) ions it is not surprising to find polynuclear iron(III) species. The first two examples of polynuclear iron(III) discussed in chapter 21, phosvitin and gastroferrin, have been implicated ${ }^{97,98}$ in the regulation and inhibition of iron absorption. The egg protein phosvitin has been suggested also to act as an iron carrier during the development of the chick embryo ${ }^{99}$. Furthermore, gastroferrin possesses bloodgroup activity, at a level similar to that of purified blood-group substance from ovarian cysts, and blood-group active substances have been reported to bind iron(III) ${ }^{98}$.

By virtue of their biological function, storage forms of iron are expected to be characterised by a high iron: protein ratio, and consequently by polynuclear interaction among the bound iron(III) ions. The inner iron core of the storage protein ferritin (section 21.3) can attain a molecular weight comparable to that of the polypeptide chains of the protein ${ }^{100}$. This storage iron appears as a discrete compact sphere of polymeric iron(III), which is maintained in a soluble biologically available form by a surrounding layer of polypeptide. The second storage form of iron considered in chapter 21 , haemosiderin, also stores iron in a spatially compact form $^{101}$. The general morphology of ferritin appears again in the final example of biological polynuclear iron, iron-dextran ${ }^{102}$, the primary function of which seems to be to provide iron for an organism. 